\title{
RECONHECIMENTO DE FALHAS ESTRUTURAIS UTILIZANDO SISTEMA IMUNOLOGICO ARTIFICIAL WAVELET
}

\section{STRUCTURAL FAILURE RECOGNITION USING SYSTEM IMMUNOLOGICAL ARTIFICIAL WAVELET}

Fábio Roberto Chavarette ${ }^{1}$, Roberto Outa ${ }^{2}$, Igor Feliciani Merizio ${ }^{1}$, Thiago Carreta Moro ${ }^{1}$, Simone Silva Frutuoso de Souza ${ }^{4}$, Fernando Parra dos Anjos Lima $^{5}$

${ }^{1}$ Universidade Estadual Paulista Júlio de Mesquita Filho - UNESP, ${ }^{2}$ Faculdades de Tecnologia do Estado de São Paulo - FATEC, Araçatuba, SP, ${ }^{4}$ Universidade do Estado de Mato Grosso - UNEMAT, ${ }^{5}$ Instituto Federal do Mato Grosso - IFMT.

E-mail: fabio.chavarette@unesp.br

RESUMO - Este trabalho apresenta uma metodologia inteligente para o monitoramento da integridade estrutural de um rotor dinâmico, o sistema imunológico artificial Wavelet. A combinação do sistema imunológico artificial com a transformada de Wavelet gera uma ferramenta inovadora para realizar a identificação, localização e classificação de falhas estruturais. Através desta metodologia, os projetos de máquinas industriais são desenvolvidos para atender essas necessidades, reduzindo as falhas e antecipando os erros encontrados em máquinas operantes. Uma_área emergente de projetos de máquinas são as máquinas rotativas também denominadas de rotores dinâmicos, sendo estes, aplicados a turbinas de aviões, turbinas a vapor para a produção de energia elétrica, turbo compressores, entre outros. Para validar esta metodologia dados experimentais são coletados, e a partir deste, gerados diversos situações (condição normal e condições em falhas), obtendo-se uma base de dados de sinais, que foram analisados pelo método proposto. Os resultados obtidos pelo Sistema Imunológico Artificial Wavelet apresentam eficiência e robustez.

Palavras-chave: Sistemas Imunológicos Artificiais; Transformada Wavelet; Rotores Dinâmicos.

ABSTRACT - This work presents a novel approach for monitoring the structural integrity of a dynamic rotor basead in a intelligent methodology combined with a mathematical transform, the Wavelet artificial immune system. The combination of the artificial immune system with the Wavelet transform generates an innovative tool to perform the identification, localization and classification of structural failures. Through this methodology, industrial machine designs are developed to meet these needs, reducing failures and anticipating errors found in operating machines. An emerging area of machine designs are rotating machines also called dynamic rotors, which are applied to aircraft turbines, steam turbines for the production of electrical energy, turbocompressors, among others. To validate this methodology, experimental data are collected, and from this, different situations (normal condition and fault conditions) are generated, obtaining a database of signals, which were 
analyzed by the proposed method. The results obtained by the Wavelet Artificial Immune System are efficient and robust.

Keywords: Artificial Immune Systems; Wavelet Transform; Dynamic Rotors

\section{INTRODUÇÃO}

O conceito inicial da revolução industrial teve início com motores a vapor alternativos, elaborados por James Watt em 1780, considerado um sistema dinâmico rotativo e, atualmente, o conceito deste sistema é aplicado em aeronaves, veículos automotivos, motores, entre outros. Diferentes técnicas foram desenvolvidas para que, nos dias atuais, as indústrias que utilizam os sistemas rotativos possam produzir produtos de alta qualidade a baixo custo. Com a introdução do conceito da indústria 4.0, o conceito de automação em processos ficou mais evidente e forte, com isso, técnicas como structural health monitoring (SHM), non-destructive testing (NDT), statistical process control (SPC), damage prognosis (DP), entre outros, tiveram que ser introduzidas para a prognose, e deteç̧ão de danos e falhas, em estruturas dinâmicas (DERAEMAEKER, WORDEN, 2010; FARRAR; WORDEN, 2013).

Em um sistema rotativo dinâmico é possível observar dois princípios físicos, sendo um deles deslocamento lateral do eixo, e o outro o efeito giroscópio. Estes dois fenômenos são representados por um modelo matemático e podem seu comportamento podem ser demonstrado pelo conceito de vibração. Utilizando este conceito, é possível introduzir técnicas matemáticas que contribuam na análise de sinal e na prognose e detecção de falhas. 0 filtro de Wiener e a transformada de wavelets são duas técnicas que auxiliam a análise do comportamento do sinal. A introdução da bioengenharia também se faz necessária para que o sinal, a partir de que identificado, possa ser classificado e separado para que as informações do tipo de falhas e probabilidades de ocorrência possam ser identificadas. $O$ processo de integridade de um sistema pode ser analisado por um sistema de monitoramento de integridade estrutural (SHM), que consiste na observação de uma estrutura ao longo do tempo através de sensores. Assim, através das medições periódicas e intervaladas, os sinais captados pelos sensores, representam o comportamento do sistema dinâmico (DERAEMAEKER; WORDEN,
2010; GOPALAKRISHNAN; RUZZENE; HANAGUD 2011).

Diferentes pesquisadores desenvolvem pesquisas ligadas a prognose e detecção de falhas utilizando SHM, dentre eles, um dos estudos é demonstrado a aplicação da técnica de SHM para detectar falhas em rolamentos por meio de vibração, cujo resultado apresentado mostra o sinal analisado por técnicas de Fourier, janelamento de Hamming e noverlap (BURDZIK; KONIECZNY; FOLEGA, 2014). Já em um outro estudo, é demonstrado a aplicação das técnicas de condition based maintenance (CBM), structural health monitoring (SHM), e prognostics and health management (PHM) no monitoramento de vibração de rolamentos, no painel e no trem de pouso do helicóptero. 0 resultado desta pesquisa mostram que as estratégias de SHM e CM são relevantes para que ocorra a escolha correta da técnica de manutenção baseada no PHM (TINGA; LOENDERSLOOT, 2014).

Neste artigo, apresenta-se um método para realizar a análise da integridade estrutural de um rotor dinâmico utilizando o sistema imunológico artificial-Wavelet. Esta proposta é uma composição do algoritmo de seleção negativa com uma transformada matemática (MALLAT, 1999), que tem por objetivo melhorar a capacidade de diagnóstico do sistema inteligente. A combinação da transformada wavelet com o algoritmo de seleção negativa proporciona maior precisão ao diagnóstico de falhas, pois, ao decompor os sinais em vários níveis de resolução, é possível identificar as anormalidades facilmente, por causa do nível de detalhe exibido no processo de decomposição.

\section{SISTEMAS IMUNOLÓGICOS ARTIFICIAIS}

O Sistema Imunológico Artificial, representa uma classe ampla da computação inteligente que tem por princípio a implementação de algoritmos que representam metodologias de manipulação de dados, classificação, representação e raciocínio que seguem um paradigma biológico plausível: o sistema imunológico humano. Assim tais algoritmos configuram ao SIA a capacidade e 
sucesso na implementação (LIMA et al., 2017; FARRAR; WORDEN, 2013; MORO et al., 2019).

Circunscrito pelo SIA, o algoritmo de Seleção Negativa proposto por (FORREST et al, 1994), baseia-se no processo de seleção que ocorre no interior do Timo. Este processo trabalha na diferenciação das células próprias e não próprias do corpo humano (LIMA et al., 2017). Assim o ANS apresenta a característica de detectar mudanças em estados de sistemas segmentado em duas fases de funcionamento: Censoriamento e Monitoramento

\subsection{Censoriamento}

$\mathrm{Na}$ fase de Censoriamento do ASN, são definidos os detectores próprios, conhecidos também como cadeias próprias (S), representam a condição normal do problema. Em seguida, é produzido o conjunto de detectores $(R)$, que possuem a capacidade de reconhecer padrões não próprios. Portanto, com a leitura dos dados, verifica-se a afinidade de cadeias aleatórias, comparando estas cadeias ao conjunto de cadeias próprias.

Se a afinidade estiver acima de um limiar preestabelecido a cadeia é rejeitada. Entretanto se a afinidade estiver abaixo do limiar, à cadeia é adicionada ao conjunto de detectores, denominado também por Base-line, sendo utilizada na classificação durante 0 monitoramento dos dados.

\subsection{Monitoramento}

$\mathrm{Na}$ fase de monitoramento, é realizado o monitoramento dos dados com o objetivo de identificar anomalias de comportamento das amostras. Assim, com o conjunto de detectores, também denominado de Matriz de verificação oriundo da fase de Censoriamento, classificar esta anomalia.

Deste modo, comparando as cadeias protegidas $(S)$ com o conjunto de detectores $(R)$, afere-se a afinidade entre as cadeias. Caso a afinidade seja superior a um limiar preestabelecido, e identificado e classificado um elemento não próprio.

\subsection{Critério de Casamento e taxa de afinidade.}

O critério de casamento ou combinação, segundo (FORREST; PERELSON; CHERUKURI, 1994), é utilizado para avaliar afinidade entre as cadeias (antígeno e anticorpo) e comprovar se estas são semelhantes ou iguais. Este casamento pode ser perfeito ou parcial.
Se o casamento for perfeito, significa que as duas cadeias analisadas apresentam os mesmos valores e logo ambas devem ser perfeitamente iguais. Entretanto, se apenas uma quantidade de posições entre os padrões possuírem o mesmo valor este casamento é definido como parcial. Neste caso, a quantidade de posições iguais obedece a um limiar preestabelecido e denominado taxa de afinidade.

Conforme (LIMA et al., 2017), o casamento perfeito entre as cadeias e dados é quase impossível de ser alcançado experimentalmente, devido as inúmeras variáveis que permeiam e alteram os dados empíricos. Por isso a utilização do casamento parcial e a taxa de afinidade mostram-se muito mais vantajosos.

A taxa afinidade utilizada como o grau de semelhança necessário para que ocorra o casamento parcial, avaliada para os números reais, é definida, de acordo com (LIMA et al, 2017) como $T A_{f}=\left(A_{n} / A_{t}\right) / 100$ sendo $T A_{f}$ a taxa de afinidade; $A_{n}$ o número de cadeias normais no problema (cadeias próprias) e $A_{t} 0$ número total de cadeias no problema (cadeias próprias e não-próprias).

Além da Taxa de afinidade, para analisar os padrões de cadeia é utilizado a Afinidade total, definida como:

$$
A f_{t}=\frac{\sum_{i=L}^{N} V_{c}}{L} 100
$$

sendo $A f_{t}$ a porcentagem de afinidade entre os padrões analisados; $L$ a quantidade total de variáveis; $V_{c}$ as variáveis casadas e $\sum_{i=L}^{N} V_{c}$ a somatória (quantidade) de variáveis casadas.

\section{TRANSFORMADA WAVELET}

As funções wavelets constituem-se em transformadas matemáticas capazes de decompor funções, permitindo reescrever estas funções com os mais finos detalhes, ou seja, permite uma visão "global".

Desta forma, podem-se diferenciar características locais de um sinal em diferentes escalas (resoluções) e, ainda, analisar toda a extensão do sinal por meio de translações. Como a maioria das wavelets possuem suporte compacto, são muito úteis na análise de sinais não-estacionários. Neste sentido, a análise wavelet leva vantagem em relação à análise de Fourier (MALLAT, 1999).

Existem diversas famílias de funções wavelet. Neste trabalho, consideram-se as 
funções wavelets ortonormais da família discretas de Daubechies (DAUBECHIES, 1992) por possuírem transformadas com algoritmos computacionalmente rápidos (MALLAT, 1999).

\subsection{Transformada Wavelet Discreta (TWD)}

Dado um sinal $y[t]=\left(y_{0}, \ldots, y_{n-1}, y_{n}\right)$ representando por um vetor discreto, pode ser representado por uma série wavelet como abaixo, (MALLAT, 1999):

$$
y[t]=\sum_{k=0}^{N_{J}} c_{J, k} \phi_{J, k}(t)+\sum_{j=J}^{1} \sum_{l=0}^{N_{j}} d_{j, k} v_{j, k}(t), \nabla t \in\left[0, N_{0}\right] \text { (2) }
$$

em que J representa o nível de resolução, $N_{j}=(N / 2)-1$ representa o número de pontos de cada novo vetor obtido pela transformação, $\phi_{j, k}(t)$ e $v_{j, k}(t)$ são as funções escala e wavelet, responsáveis pela transformação; $j$ indica escala (dilatação) e $k$ a posição (translação).

A Transformada Wavelet Discreta (TWD) aplicada diretamente a sinal para a geração do conjunto de coeficientes é computada por sucessivas passagens pelo filtro $G$ (passa baixa) e pelo filtro $H$ (passa alta), ou como também conhecido níveis de resolução. Os filtros $G$ e $H$ são vetores de constantes já calculados que proporcionam uma base ortogonal relacionada às funções escala e wavelet, respectivamente. Esse processo é conhecido como algoritmo Piramidal de Mallat (MALLAT, 1999) é ilustrado na Figura 1.

Figura 1. Representação do algoritmo piramidal para TWD.

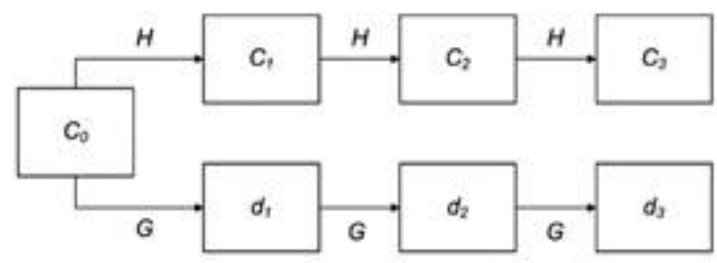

Fonte: (Mallat, 1999).

Na Figura 1, $C_{0}$ corresponde ao sinal original discreto $\left(C_{0}=y[t]\right), H$ e $G$ representam os filtros passa-alta e passa-baixa, respectivamente. Os parâmetros $d_{1}, d_{2}$ e $d_{3}$ são os coeficientes wavelets ou detalhe em cada nível de resolução e $C_{3}$ são os coeficientes escala ou aproximação no último nível de transformada. Estes coeficientes são obtidos através da convolução das constantes nos filtros (3) e (4), (MALLAT, 1999):

$$
\begin{gathered}
C_{j+1, k}=\sum_{l=0}^{D-1} h_{l} C_{j, 2 k+l} \\
d_{j+1, k}=\sum_{l=0}^{D-1} g_{l} C_{j, 2 k+l}
\end{gathered}
$$

sendo $k=\left[0, \ldots,\left(N / 2^{j}\right)-1\right]$ e $D$ o número de constantes do filtro. Desta forma, os coeficientes $C_{j, k}$ representam a média local ponderada e os coeficientes wavelet $d_{j, k}$ representam a informação complementar ou simplesmente os detalhes que escapam da média ponderada. Assim, os coeficientes da transformada ordenados por escala (j) e posição (k) e são representados como a seguir (MALLAT, 1999):

$$
\psi=\left(\left(C_{J, k}\right)_{k=0}^{N_{J}},\left(\left(d_{j, k}\right)_{k=0}^{N_{J}}\right)_{j=J}^{1}\right)
$$

de forma que $\psi$ é a representação finita apenas em termos dos coeficientes da decomposição do sinal na Equação (5).

\section{BANCADA EXPERIMENTAL}

Para o desenvolvimento da metodologia proposta é necessário inicialmente, apresentar a descrição da bancada experimental com a finalidade de obter um conjunto de dados que foram utilizados para aplicação no monitoramento de falhas estruturais mecânicas.

O modelo experimental contém um motor elétrico acoplado em uma polia, e atrelado a esta polia está uma correia atrelada à outra polia, ligada a um eixo. Este eixo é apoiado em dois mancais de rolamentos. As especificações dos equipamentos são: um motor de $0,5 \mathrm{cv} ; 60 \mathrm{~Hz}$; o inversor de frequência tem capacidade de atuar na frequência de 0 a $60 \mathrm{~Hz}$; o material do eixo é aço 1045 de diâmetro de $12 \mathrm{~mm}$; a distância entre eixos de $128 \mathrm{~mm}$; dois mancais de rolamento P205 UC205 e um mancal de rolamento usado P205 UC205. O equipamento utilizado para a medição da vibração atua na faixa de frequência de $10 \mathrm{~Hz}$ a $1 \mathrm{KHz}$, com sensor de vibração de sensibilidade aproximada de $15 \mathrm{~mA} D C$, cujos requisitos dos instrumentos para a medição de intensidade da vibração em máquinas rotativas $\mathrm{e}$ alternativas satisfazem a norma ISO2954. Figura 2 a seguir apresenta o equipamento utilizado para a captação dos dados. 
Figura 2. Bancada Experimental - Rotor Dinâmico

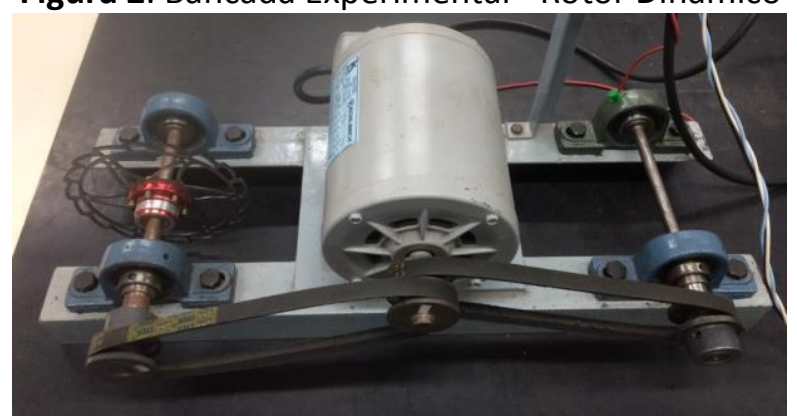

Fonte: Próprio autor.

Dados experimentais são coletados para a análise de comportamento de falhas do rotor dinâmico adotando-se o comportamento normal (sem falhas) e, fazendo a frequência variar de $4 \mathrm{~Hz}$ a $60 \mathrm{~Hz}$, com passo de $2 \mathrm{~Hz}$, gera-se um banco de dados (baselines) de sinais que recebem a denominação de Sinais Próprios. A partir da introdução de falhas (massas desbalanceadoras), captaram-se novos sinais denominados de Sinais Não-Próprios gerando-se assim um banco de dados completo para a aplicação da metodologia proposta.

\section{METODOLOGIA PROPOSTA}

O sistema de análise da integridade estrutural proposto neste artigo consiste-se em quatro módulos principais: aquisição de dados, transformada Wavelet, censoriamento e monitoramento do sistema imunológico artificial.

O módulo de aquisição de dados é constituído pelo aparato experimental responsável por capturar os sinais na estrutura do rotor dinâmico, tais como sensores, acelerômetros, etc. Os sinais representam a resposta em frequência, resultante da FFT obtida no módulo de entrada. Além disso, os sinais são processados pelo módulo de transformada de Wavelet. O conjunto de sinais obtidos no domínio Wavelet são usados como entradas para sistema imunológico artificial.

Módulo de Censoriamento do ASN: O sistema de diagnostico foi executado em duas fases, sendo uma fase off-line e outra on-line. No processo off-line foi executado o aprendizado (treinamento) inicial do sistema, denominado de modulo de censoriamento. Neste processo, foram definidos os detectores próprios, formando o conjunto de detectores do ASN. Este conjunto de detectores foi o conhecimento do sistema inteligente, para tomada de decisões na fase on-line.
O conjunto de detectores próprios constituídos de 46 sinais que possuem a característica normal da estrutura, ou seja, sem danos (baseline) e 120 sinais que possuem as características dos danos, ou seja, as situações distintas de danos.

Neste contexto, foi gerado o conjunto de detectores próprios escolhidos aleatoriamente entre sinais normais e sinais com danos não repetidos no banco de dados. Os dados foram comparados, ponto a ponto, com os detectores próprios. Se um casamento foi encontrado, o vetor aleatório foi rejeitado, pois foram constatadas características próprias. Caso contrário, foi aceito, sendo armazenado como detector próprio no conjunto de detectores próprios.

Após o conjunto de detectores obtido, o mesmo foi utilizado nas etapas do sistema de diagnostico, tais como: o módulo de monitoramento do ASN. Depois de realizar o processo em modo on-line foi executado o processo de monitoramento em modo on-line.

Módulo de Monitoramento do ASN: O modulo de monitoramento é dividida em três etapas: módulo de entrada ou leitura dos sinais (via sistema de aquisição de dados), módulo de Wavelet, responsável por aplicar a transformada de Wavelet, módulo de deteç̧ão, responsável por realizar a discriminação próprio/não-próprio, identificando as danos, e, finalmente, o módulo de classificação, responsável por caracterizar as danos estruturais.

\section{RESULTADOS}

Após a montagem da bancada experimental foi introduzido no experimento, duas massas para o desbalanceamento do sistema, considerado falhas, sendo esta introdução, intercalada uma após a outra, e as duas simultâneas consecutivas. Nestes três casos: experimento sem massa, introdução de uma massa, introdução de duas massas, foram captados os sinais de cada fase e tratados separadamente. Foi montado um segundo banco de dados com as mesmas características do primeiro banco de dados: aquisição da vibração de $4 \mathrm{~Hz}$ a $60 \mathrm{~Hz}$, a cada $2 \mathrm{~Hz}$ com os dois mancais novos. A amostragem a cada $2 \mathrm{~Hz}$, chamada de sinal, cada sinal é composto de 400 amostras de aceleração. O resultado da coleta de dados experimentais foram duas matrizes de $46 \times 400$ sinais sem falhas e $120 \times 400$ sinais com falhas. 
Após o tratamento dos sinais, o algoritmo atua analisando os sinais nas fases de censoriamento e monitoramento, e utilizando o critério de casamento e afinidade para cada matriz de sinal sem falhas e a outra com falhas, respectivamente. $O$ resultado da aplicação do algoritmo dos sistemas imunológicos artificiais utilizando reconhecimento de padrões analisou 166 sinais com 400 amostras, e detectou que 46 dos sinais não contêm falhas, ou seja, identificação e reconhecimento assertivo de $100 \%$ das amostras de sinais sem falhas.

\section{CONSIDERAÇÕES FINAIS}

Neste trabalho foi proposta uma nova metodologia para análise de integridade estrutural de um rotor dinâmico, em que se empregou o sistema imunológico artificial Wavelet. Os resultados apresentados mostram sua excelência obtendo um índice de acerto de $100 \%$ para a melhor configuração do sistema. Esta proposta é uma composição do sistema imunológico artificial com a transformada Wavelet, que tem por objetivo melhorar a capacidade de diagnóstico do sistema inteligente. Tal proposta demonstrou ter uma excelente funcionalidade e coerência. Por fim, conclui-se que o sistema imunológico artificial Wavelet proposto é eficiente, confiável e robusto na análise de integridade de estruturas mecânicas.

\section{AGRADECIMENTOS}

Agradecemos ao Laboratório de Sistemas Complexos (Sisplexos) da Faculdade de Engenharia de Ilha Solteira - UNESP por ceder o espaço físico e o parque computacional e ao suporte financeiro oferecido pela Fundação de Amparo à Pesquisa (FAPESP), através do processo 2019/10515-4 e ao Conselho Nacional de Desenvolvimento Científico e Tecnológico (CNPq), através do processo 312972/2019-9.

\section{REFERÊNCIAS}

BURDZIK, R.; KONIECZNY, t.; FOLEGA, P. Structural Health Monitoring of Rotating Machines in Manufacturing Processes by Vibration Methods, Advanced Materials Research, v. 1036, n. 1, p. 642-647, 2014. Disponível em: http://dx.doi.org/10.4028/www.scientific.net/am r.1036.642. Acesso em : 25 jun.2020. https://doi.org/10.4028/www.scientific.net/AMR .1036 .642
DAUBECHIES, I. Ten Lectures on Wavelets. Philadelphia: Society for Industrial and Applied Mathematics, 1992.https://doi.org/10.1137/1.9781611970104

DERAEMAEKER, A.; WORDEN, K. New trends in vibration based structural health monitoring. New York: SpringerWien, 2010. https://doi.org/10.1007/978-3-7091-0399-9

FARRAR, C. R.; WORDEN, K. Structural Health Monitoring: A Machine Learning Perspective. Chichester: John Wiley, 2013. https://doi.org/10.1002/9781118443118

FORREST, S. A.; PERELSON, A. L.; CHERUKURI, R. Self-nonself discrimination in a computer. In: IEEE SYMPOSIUM ON RESEARCH IN SECURITY AND PRIVACY. 1994. Oakland. Proceedings [...].Oakland: IEEE, 1994, p. 202-212.

GOPALAKRISHNAN, S.; RUZZENE, M.; HANAGUD, $S$. Computational techniques for structural health monitoring. London: Springer-Verlag, 2011, p. 517. https://doi.org/10.1007/978-085729-284-1

LIMA, F. P. A.; CHAVARETTE, F. R.; SOUZA, S. S. F.; LOPES, M. L. M. Monitoring and Fault Identification in Aeronautical Structures Using an Wavelet-Artificial Immune System Algorithm. In: EKWARO-OSIRE, S. et al. , (org.). Probabilistic Prognostics and Health Management of Energy Systems. 12 ed. São Paulo: Springer International Publishing AG, Chaim, 1, 2017, p. 1-17.

MALLAT, S. A Wavelet tour of signal processing, New York: Academic Press, 1999. https://doi.org/10.1016/B978-012466606$1 / 50008-8$

MORO, T. C.; CHAVARETTE, F. R., ROEFERO, L. G. P., OUTA, R. Detecão de falhas estruturais de um prédio de dois andares utilizando Sistema Imunológico Artificial. Colloquium Exactarum, v. 11, p. 73-84, 2019. Disponível em: http://dx.doi.org/10.5747/ce.2019.v11.n4.e298. Acesso em: 25 jun. 2020. https://doi.org/10.5747/ce.2019.v11.n4.e298

TINGA, T.; LOENDERSLOOT, R. Aligning PHM, SHM and $\mathrm{CBM}$ by Understanding the Physical System Failure Behaviour, In: EUROPEAN CONFERENCE OF THE PROGNOSTICS AND HEALTH 
MANAGEMENT SOCIETY. 2. , 2014. Nantes.

Proceedings [...] Nantes, 2014 v. 2, n. 1, p. 162-

171, 